\title{
Tipping points in the climate system
}

\author{
Peter Ditlevsen
}

August 26, 2015

\begin{abstract}
In climatic time series abrupt changes are observed. We hypothesize that these abrupt changes are due to nonlinear responses inherent in the climate system, specifically, so-called tipping points.

This behaviour result from non-linear climate response to either external forcing, internal stochastic fluctuations or a combination of both. At some point the forcing will cause the climate to jump from one stable state to another. This scenario is termed a tipping point. The concept of a tipping point is quite broad, but here we shall refrain from any general definitions and consider the following more restricted framework: We consider the climate or some components of the climate as a dynamical system depending on a set of parameters. Factors, not included in the system interacting with components of the system, can then be considered external forcing or stochastic fluctuations. Two common, and often competing, hypotheses are: The climate system's steady state loses its stability and disappears as an external system (control-)parameter slowly changes, so-called b-tipping, b for bifurcation-induced; or fluctuations spontaneously push the climate system from one stable state to another, so-called n-tipping, $\mathbf{n}$ for noise-induced.

The cause of the tipping can be very different in the two cases, and especially the possibility of predicting a tipping will be different. In the case that the underlying dynamics or the control-parameter are not completely known, there could still be early warning signals in the statistics of the observed fluctuations prior to a tipping point.
\end{abstract}

The Pleistocene climate is documented in a variety of geological records, most promi- 


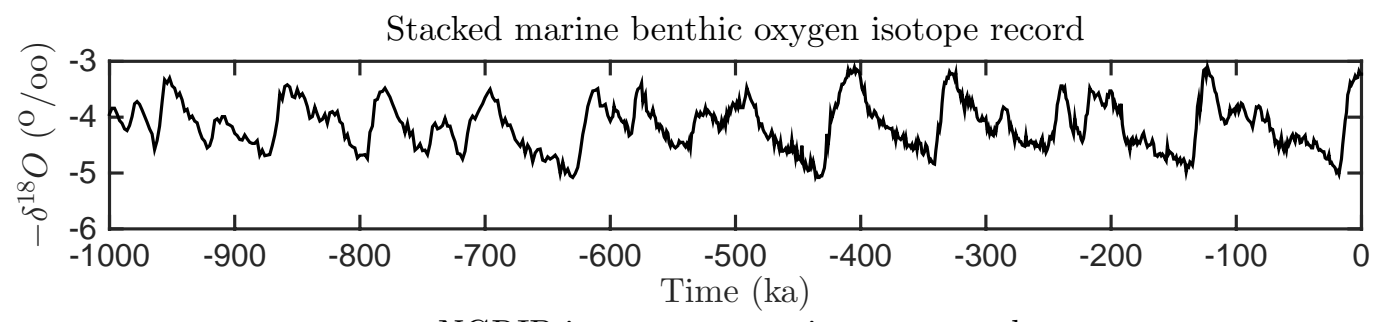

NGRIP ice core oxygen isotope record

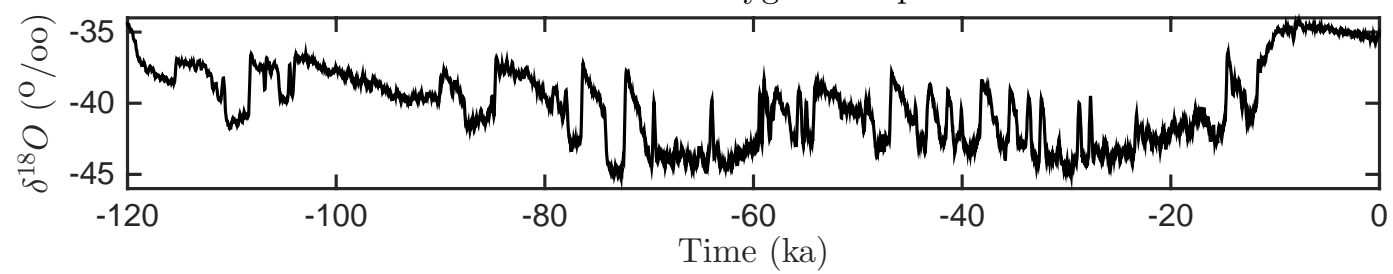

Figure 1: The top panel shows the last $1 \mathrm{Myr}$ of the Lisiecki and Raymo stack marine benthic oxygen isotope record (Lisiecki \& Raymo, 2005). The record is a proxy for global ice volume, showing the last ten glacial cycles. The bottom panel shows the NGRIP isotope record (North GRIP members, 2004) of the last glacial period. The glacial climate was dominated by rapid fluctuations between cold and warmer periods, the so-called Dansgaard-Oeschger events. The minus in front of $\delta^{18} O$ is such that for both panels have increasing paleo temperatures upward.

nently in ocean sediment cores and ice cores. These all show that climate has changed abruptly through time both as response to the orbital changes and as a part of internal variability. Figure 1, top panel, shows the last $1 \mathrm{Myr}$ of the Lisiecki and Raymo stack marine benthic oxygen isotope record (Lisiecki \& Raymo, 2005). The record is a proxy for global ice volume, showing the last ten glacial cycles. There is a strong and consistent time asymmetry with rapid warming (terminations) and gradual cooling (inceptions). The time asymmetry is not present in the orbital changes (Berger, 1978), indicating that the climate response to the orbital forcing is non-linear. The terminations are hypothesised to be tipping points, where the insolation crosses a threshold such that the mass balance of the Northern ice sheets cannot be maintained (Weertman, 1961; Paillard, 1998). The bottom panel shows the NGRIP isotope record (North GRIP members, 2004) of the last glacial period. This is a temperature proxy, showing much stronger variability during the glacial period than in the interglacial. Most pronounced are the Dansgaard-Oeschger (DO) events (Dansgaard et al., 1993), which have no known external trigger. 


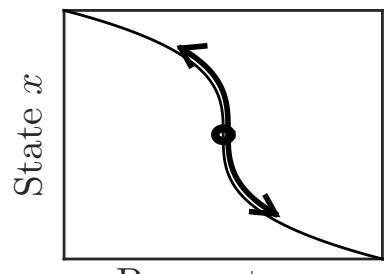

Parameter $\mu$

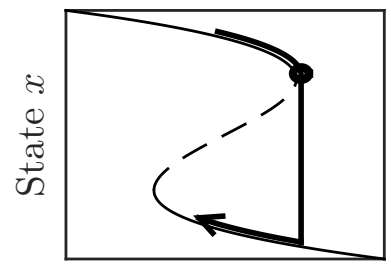

Parameter $\mu$

Figure 2: Schematic graphs of steady states $x$ as a function of a control parameter $\mu$. In the left panel there is only one steady state solution and changes in the state are reversible as the parameter is charged. In the right panel multiple solutions exist and changes are irreversible if a bifurcation point is crossed. In order to return to the upper state again in the case shown in the right panel, the parameter must change to a lower value where another bifurcation point is reached. In both cases the circles indicate where $d x / d \mu$ diverge locally. The thick curves (arrows) indicate the steady state as $\mu$ is increased and subsequently decreased.

\section{Irreversibility and the feedback factor}

The definition of the term "tipping point" in the climate system is somewhat vague, describing the general feature that a change in some parameter $\mu$ in the system, often termed a control parameter, can lead to a disproportionately large change in the state of the system. Such a change can be reversible in the sense that changing the control parameter back to the original value will reset the system to the original state. Otherwise, the system can change irreversibly to a different state, such that resetting the control parameter does not make the system return to its original state. This latter possibility implies the existence of multiple steady states of the system.

The two different scenarios are shown schematically in figure 2. The solid curves represent stable steady states, while dashed the curve in the right panel is the separating unstable steady state. In the left panel a change of the control parameter $\mu$ leads to a large, but reversible change in state, while in the right panel the system undergoes a bifurcation leading to an irreversible change of state. Distinguishing these two situations requires an analysis beyond the linear response. To illustrate that consider the system governed by the equation

$$
\dot{y}=f(y, \mu) .
$$

The response $\Delta x$ to a change $\Delta \mu$ is described in terms of the linear feedback factor $\lambda=d x / d \mu: \Delta x=\lambda \Delta \mu$. The feedback factor is obtained from the governing 
equation, with $x$ being a steady state, $0=\dot{x}=f(x, \mu)$. We thus have

$$
\begin{aligned}
0 & =f(x+\Delta x, \mu+\Delta \mu) \approx f_{x}(x, \mu) \Delta x+f_{\mu}(x, \mu) \Delta \mu \\
& \Rightarrow \lambda=\Delta x / \Delta \mu=-f_{\mu} / f_{x}
\end{aligned}
$$

In both cases illustrated in figure 2 we get $\lambda \rightarrow-\infty$ as the points marked with circles are approached. However, the two situations are completely different, the second is in this context an irreversible tipping point, where the system goes through a bifurcation, while the first is not.

\section{Bifurcation theory}

Before continuing investigating transitions in the climate, we shall describe the most elementary basics of bifurcation theory (Ma \& Wang, 2005). Bifurcation theory describes the behaviour of a dynamical system governed by (1) when there is a qualitative change in the set of solutions to (1), as function of initial values, when the control parameter $\mu$ is continuously changed through a critical value. To make this statement concrete, consider the situation depicted in the right panel in figure 2: For values of $\mu$ to the right of the bifurcation point (the circle) all solutions regardless of initial value will end up on the lower branch. On the contrary when $\mu$ is on the left side of the bifurcation point solutions initially below the dashed line will end up on the lower stable branch, while solutions initially above the dashed line will end up on the upper branch. This "forking out" from one to two solution is the motivation for the technical term "bifurcation".

When we only consider the structural change of dynamics close to the bifurcation point in the phase space (which in this case is the space along the y-axis) this is a local bifurcation. One virtue of local bifurcation theory is that for a single control parameter and a continuous dynamics, that is $f(y, \mu)$ is a continuous function of $y$ and $\mu$, then there is a very small universal list of possible bifurcations. If we further consider generic behaviour in the sense that perturbing $f(y, \mu)$ slightly will not change the behaviour, then we are left with only two possibilities, the saddlenode bifurcation, which is the one shown by the circle in the second panel in figure 2. The only other generic bifurcation is the Hopf bifurcation, where a single steady state turns into a periodic oscillation (a limit cycle) at the bifurcation point.

Here we shall only be concerned with the saddle-node bifurcation. Close to the bifurcation point the dynamics will be topological equivalent to the one dimensional dynamics in the so-called normal form, where $x$ is now a single variable:

$$
\dot{x}=-x^{2}-\mu
$$


The fixed points are $x_{ \pm}(\mu)= \pm \sqrt{-\mu}$. Thus only for $\mu<0$ will there be fixed points, for $\mu>0$ the right hand side of (3) is always negative and $x$ will escape to $-\infty$ or rather to some other state outside the domain where (3) is applicable. The stability of the fixed points is determined from the linearised equation for perturbations around the fixed point, $\xi_{ \pm}=x-x_{ \pm}$:

$$
\dot{\xi}_{ \pm}=\mp 2 \sqrt{-\mu} \xi_{ \pm}
$$

thus $x_{+}(\mu)$ is the stable branch, where perturbations decay exponentially (curve of stable fixed points as a function of $\mu$ ), while $x_{-}(\mu)$ is the unstable branch, where perturbations grow exponentially. The two branches merge at the bifurcation point $x=0$. 

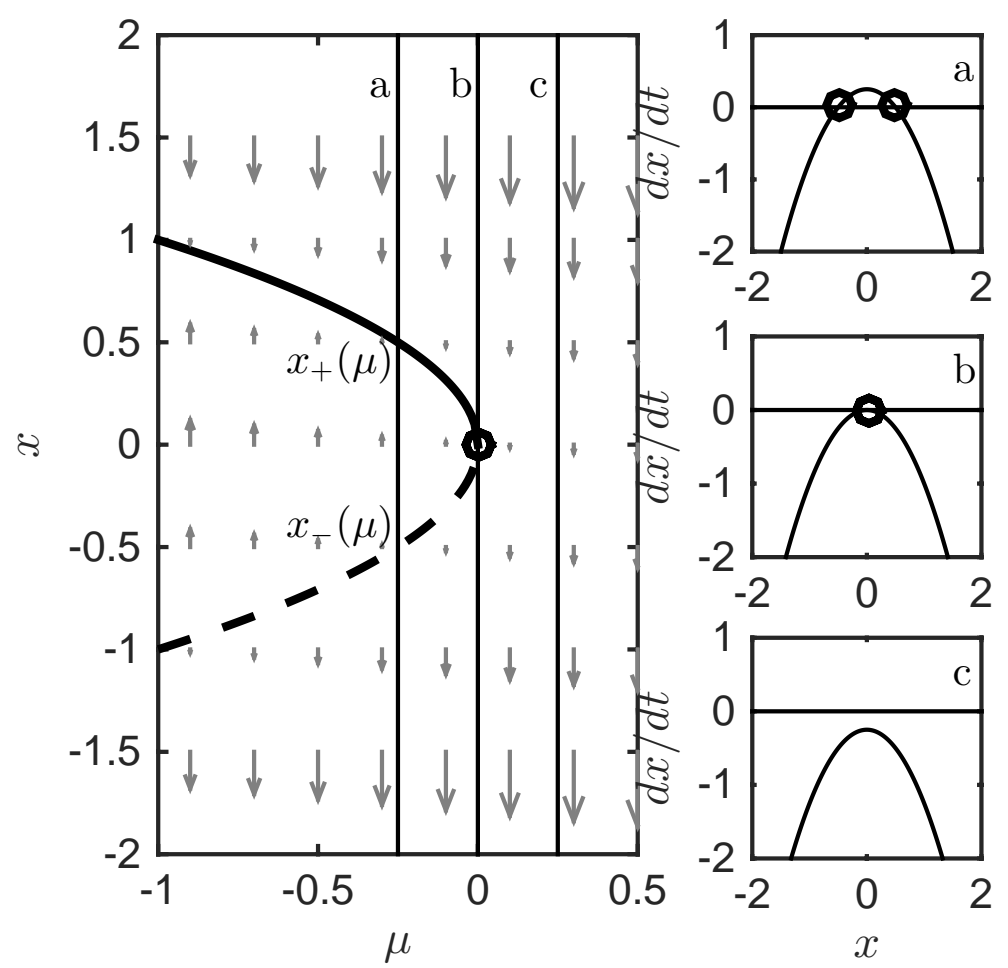

Figure 3: The left panel shows the bifurcation diagram near a saddle-node bifurcation. The top branch, the node (full curve) $x_{+}(\mu)$ is stable while the bottom branch, the saddle (dashed curve) $x_{-}(\mu)$ is unstable. The grey arrows indicate the flow in phase space governed by equation (4) for constant values of the control parameter $\mu$. The circle marks the bifurcation point. The panels at the right shows $d x / d t$ along the three vertical lines in the left panel. Crossings of the x-axis, marked with circles, indicate fixed points. For $\mu<0$ there are two fixed points, which merge at $\mu=0$. For $\mu>0$ there are no fixed points. 
Figure 3, left panel, shows the bifurcation diagram of the generic saddle-node bifurcation. The full line is the stable branch, while the dashed line is the unstable branch. The bifurcation point is marked by a circle. The vector field $(\dot{\mu}=0, \dot{x})$ is shown in grey. To see this clearer, the right panels in Figure 3 show the derivative $\dot{x}$ along the three vertical lines in the left panel. Steady states are where the parabola crosses the x-axis. The term saddle-node bifurcation simply comes from the merger of the unstable, "saddle" fixed point and the stable, "node" fixed point.

The climate system has many degrees of freedom, and operates over many time scales. We may assumed that the phenomenon in question can be described by a set of variables $x_{i}=x_{1}, \ldots, x_{N}$, where $N$ need not be a small number, and a set of governing equations,

$$
\dot{x}_{i}=F_{i}\left(x_{1}, \ldots, x_{N}, \mu\right) .
$$

The reason why the high dimensional climate dynamics can be reduced to a single variable representation (1) is that varying the single parameter $\mu$ maps out the (one dimensional) stable fixed point curve $x_{i}^{0}(\mu)$, where $F_{i}\left(\mathbf{x}^{0}\right)=0$. The stability of the fixed point with respect to perturbations is determined from the linear stability analysis; $\dot{\xi}_{i}=J_{i j} \xi_{j}$, where $J_{i j}=\left(\partial F_{i} / \partial \xi_{j}\right)_{\mathbf{x}=\mathbf{x}^{0}}$ is the Jacobian. Since $x_{i}^{0}(\mu)$ is the stable branch, all eigenvalues of $J_{i j}$ have negative real parts. When crossing the generic saddle-node bifurcation a single real eigenvalue $\lambda(\mu)$ will become positive. The corresponding eigenmode $\xi_{i},\left(\dot{\xi}_{i}=J_{i j} \xi_{j}=\lambda \xi_{i}\right)$ will grow exponentially. This represents the most unstable direction in phase space. The other case of a pair of complex conjugate eigenvalues crossing the imaginary axis corresponds to a Hopf bifurcation, which we shall not consider here.

\section{Multiple states in the climate}

In the climate system, we do not have a full understanding of components with the potential of tipping. An overview, as some sort of expert assessment, has been proposed (Lenton et al., 2008). In our context tipping is associated with existence of multiple states, as shown in the second panel in figure 2. A few prominent examples of possible multiple states in the climate can be described in terms of simple dynamical systems: The global energy balance models (Budyko, 1969; Sellers, 1969) can be simplified to a single equation for the mean surface temperature, balancing incoming short wave and outgoing long wave radiation. The planetary albedo, governing the reflection depends on the ice-albedo feedback, such that there are two possible stable steady states: An ice covered cold planet, with high reflection and low long 
wave outgoing radiation and a warm planet with high absorption and high outgoing long wave radiation. The control parameter $\mu$ forcing a bifurcation from one to the other state could be the atmospheric greenhouse gas concentration, permitting high surface temperature with lower outgoing radiation emitted from the top of the atmosphere, or it could be the insolation changing the total incoming short wave radiation. It is worth noting that even though the global energy balance models did not explain the glacial cycles, they actually predicted the existence of the Snowball Earth climate (Hoffman et al., 1998), which was not known when the models were proposed. This is thus one of very few theoretical predictions in climate theory.

The Atlantic meridional overturning circulation (AMOC) was explained in the very simple Stommel (Stommel, 1961) two box hydrological link model. The thermohaline circulation is forced by buoyancy with heavy surface water sinking to the bottom, driving the meridional overturning circulation. The surface water can be heavy because it gets cold from cooling to the atmosphere or because it gets saltier due to fresh water being evaporated from the surface. For a given heating/evaporation the model has two stable steady states of the flow: The on-mode where the sinking, deep water formation, happens in the northern ocean because the salty surface waters cools as it moves north. In the other state, the off-mode, the evaporation in the warm equatorward surface waters and the precipitation into the poleward surface waters leaves the heavy water to sink in the south. The Stommel box-model is extremely simple, but it captures the essential multi state dynamics also found in numerical ocean models solving the governing fluid mechanics equations (Rahmstorf, 1995). The model has a set of bifurcations from one to three steady states, where in the latter case the two stable steady states are the on- and off-modes. In this case the control parameter $\mu$ is typically the fresh water input into the mid latitude band of the ocean.

Ice sheet dynamics might play an important role in glaciation (Weertman, 1961). The size of an ice sheet is determined by the mass balance between accumulation of snow and melting at the margin. The central part of the ice sheet where growth dominate is called the accumulation zone, the temperature is below freezing either because it is more poleward or because it is at high altitude. The marginal part where melting and ice berg break off dominates is called the ablation zone. The steady state shape of the ice sheet is maintained by ice flow from the accumulation to the ablation zone. For given atmospheric temperature and precipitation fields multiple states of the ice sheet could exist, namely a grown ice sheet, where the altitude of the central ice sheet makes it cold enough to maintain a sufficient accumulation zone. Alternatively, if the ice sheet is to low, the accumulation zone is to small to maintain mass balance and the ice sheet collapse into the other stable state of no ice sheet at 
all. The control parameter for this system could be the summer temperature at the equator-ward rim of the ice sheet.

For the most pronounced climate oscillation we observe, the glacial cycles, we do not have a full theory yet. There are multiple lines of evidence that the Milankovitch theory is essentially correct (Hays et al., 1976). The glacial cycles are related to variations in Earth's orbit around the Sun and the tilt of Earth's axis of rotation with respect to the plane of the orbit. From the climate record it is also evident that climatic response to the orbital forcing is not linear. The climate record is time a-symmetric, while the forcing is time symmetric. The record thus suggests that the climate is jumping between different stationary states. If the dynamics is dominated by waxing and waning of the Northern hemisphere ice sheets, the control parameter could be the $65 \mathrm{~N}$ summer insolation as suggested by Milankovitch (Milankovitch, 1930).

Finally, the coupling between the vegetation and the climate can also be modelled as a two state system, with green moist planet, where the vegetation provides evapotranspiration and cooling to maintain suitable growth conditions and a dry and hot desert state where vegetation cannot be sustained (Kleidon et al., 2000). In this case the control parameter could be local precipitation or temperature.

\section{The effective dynamics}

When considering specific climatic phenomena, or when seeking the physical explanations for the variations observed in climatic and paleoclimatic time series, it is often useful to keep the Global Climate Models, or Earth System Models (ESM), in mind as the paradigm for our present understanding. The basic assumption of these models are that the evolution of the climate can be simulated by numerical integration the Navier-Stokes equations (albeit in a rudimentary form) for the atmospheric and oceanic flows, the heat equation, including radiative absorption and scattering, cloud processes, hydrological cycle, effective equations for interaction with ice masses, soils, vegetation etc. For the state-of-the-art models the spacial resolution, number and detail of processes incorporated is to a large extend governed by computational limitations. Though an increasing realism in ESMs, the models (still) respond quite linearly to changes in the forcing, be it changing atmospheric greenhouse gasses or orbital changes. This is in contrast to evidence from the paleoclimatic records, which document that the climate has undergone many abrupt changes, and responds strongly non-linear to the orbital forcing. The reason for this apparent discrepancy between models and observations is poorly understood. However, the structural change and the system behaviour associated with a simple bifurcation can, 
close to the bifurcation point, be categorised into one of a few possible categories, thus analysing the transitions in the observed climatic records could potentially give insight into the governing physical mechanisms.

The governing equations (5) are believed to describe the essential dynamics of the studied phenomenon. It is thus assumed that the remaining variables in the full system can be dealt with as either simply decoupled from the phenomenon, thus neglected, or as varying on time scales very different from the time scales in the equations. If these not included variables change on much slower time scales they are incorporated as parameters. If they change on much faster time scales they need to be taken into account.

Assume now, rather than by (5), the system is described by a more complete set of governing equations for the system variables that can be split in separate variables represented by the vectors $x_{i}=\left(x_{1}, \ldots x_{N}\right)$ and $y_{i}=\left(y_{1}, \ldots, y_{M}\right)$. Here we can associate a typical timescale $\tau_{y_{i}}$ and $\tau_{x_{j}}$ respectively to each variable such that $\tau_{y_{i}} \ll \tau_{x_{j}}$ for all $(i, j)$. Note that this assumption of time scale separation does not in general hold for the climate system. Several rigorous strategies have been applied (Majda et al., 2003; Papanicolaou \& Kohler, 1974). These would typically involve some expansion in a small parameter $\epsilon$, defined from the autocorrelation times: $\tau_{y_{i}}=\epsilon \tau_{x_{j}}$. Empirical comparisons show that even in the case $\epsilon \approx 1$ the strategies seems to work surprisingly well in describing observations.

For brevity we drop vector notation, and consider $x$ as a slow and $y$ as a fast scalar variable. The extension to more variables is mostly straight forward. With this the governing equations can be written

$$
\begin{aligned}
& \dot{y}=f(x, y, \mu) \\
& \dot{x}=g(x, y, \mu)
\end{aligned}
$$

where again $\mu$ is a parameter. Here we shall give a non-rigorous derivation: Equation (7) describes the dynamics of the large scale, or slow variables. In the effective dynamics for $x$ we can write the fast variable as $y=\langle y \mid x\rangle+y^{\prime}$, where the brackets denote the average of $y$ conditioned on $x$. Inserting this into the second equation, using that $y$ varies much faster than $x$, we can approximate

$$
\begin{aligned}
\dot{x} & =g\left(x,\langle y \mid x\rangle+y^{\prime}, \mu\right) \approx g(x,\langle y \mid x\rangle, \mu)+\partial_{y} g(x,\langle y \mid x\rangle, \mu) y^{\prime} \\
& =g_{\mathrm{eff}}(x, \mu)+\sigma(x) \eta
\end{aligned}
$$

For the short time correlated variations $y^{\prime}(t)$ we have substituted a stochastic white noise $\eta(t)$. 
Equation (8) is called a Langevin equation. In the simplest form we will assume the noise intensity to be independent of the climate state $x$. Writing $F(x, \mu)$ for $g_{\text {eff }}(x, \mu)$ we have

$$
\dot{x}=F(x, \mu)+\sigma \eta
$$

Thus the effect of the unresolved fast variables $y$ on the variables $x$ is simply the addition of a stochastic term to (5).

A bifurcation is associated with a structural change in the stable states (fixed points) of the dynamics as one or more parameters are changed. Obviously, the climate system is never at a steady state of rest. This corresponds to (9) not having any fixed points $(\dot{x}=0)$, due to the fluctuating noise term. We should thus consider equation (5) when examining for steady state solutions. Assume that the transition away from a steady state observed is due to slow changes in a single parameter $\mu$. In the case of the AMOC this would be the freshwater forcing, in case of the global energy balance the control parameter $\mu$ could be the atmospheric greenhouse gas concentration, for glacial/interglacial transition, it could be the $65 \mathrm{~N}$ summer insolation and so on. In technical terms this is a co-dimension-one bifurcation. The generic local bifurcation in this case is the saddle-node bifurcation. Beside the Hopf bifurcation, this is the only bifurcation which is structurally stable with respect to small perturbations in the dynamics.

The reduction of the dynamics to a single effective variable represented by the most unstable direction in phase space carry the hope that essential insight into the multidimensional dynamics can be obtained from observations of a single (proxy) time series. The requirement is of course that the effective variable projects sufficiently onto the observed proxy.

\section{The stochastic dynamics}

In the bifurcation analysis above, we have neglected the effect of the stochastic noise term in (9), which we for now without loss of generality can consider to be onedimensional. If the noise intensity is low the $x$ component will be close to a stable fixed point $x_{0}$ provided $F\left(x_{0}\right)=0$ and $F^{\prime}\left(x_{0}\right)=-\alpha<0$. Note that the parameter $-\alpha$ thus is identical to the largest real eigenvalue $\lambda_{i}$ above. With a trivial shift of variables we can take $x_{0}=0$ and expand $F(x)$ in (9) to first order

$$
\dot{x}=-\alpha x+\sigma \eta
$$

A process satisfying this equation is the usual red noise process or the OrnsteinUhlenbeck process (Gardiner, 1985). A simulation of this stochastic differential equation for $x$ is shown in figure 4. As first noted by Hasselmann (Hasselmann, 1976) this 
simplest possible stochastic process gives good account for many observed climatic time series. This and the fact that all statistics for this process can be calculated analytically, makes it the natural benchmark process as the null-hypothesis for statistical testing.

For a time series $x(t)$ the autocorrelation function is defined as $c_{x}(\tau)=\langle x(t) x(t+$ $\tau)\rangle$, where $\langle$.$\rangle denotes statistical mean, which for a stationary (and ergodic) process$ is identical to the time mean of the process. From (10) a linear differential equation for the autocorrelation function $c_{x}(\tau)$ can easily be derived:

$$
\begin{aligned}
\frac{d}{d \tau} c_{x}(\tau) & =\frac{d}{d \tau}\langle x(t) x(t+\tau)\rangle \\
& =\langle x(t) \dot{x}(t+\tau)\rangle \\
& =\langle x(t)[-\alpha x(t+\tau)+\sigma \eta(t+\tau)]\rangle \\
& =-\alpha\langle x(t) x(t+\tau)\rangle+\sigma\langle x(t) \eta(t+\tau)\rangle \\
& =-\alpha c_{x}(\tau)
\end{aligned}
$$

The mean involving the product of $x(t)$ and $\eta(t+\tau)$ vanishes for $\tau>0$ because the white noise excitation $\eta(t+\tau)$, with zero mean, is future to the response $x(t)$ implying that $x(t)$ and $\eta(t+\tau)$ are independent. (In a case where $\sigma$ depends on $x$ this independence argument is only valid for the Itô interpretation of the stochastic differential equation.)

It follows from equation (10), using $\langle x(t) x(t+\tau)\rangle=\langle x(t-\tau) x(t)\rangle$, that $c_{x}(\tau)=$ $c_{0} \exp (\alpha \tau)$ for $\tau<0$, and thus

$$
c_{x}(\tau)=c_{0} \exp (-\alpha|\tau|)
$$

The factor $c_{0}=c_{x}(0)=\left\langle x^{2}\right\rangle$ is the variance of the process $x(t)$ which will be calculated shortly. The system has a typical correlation timescale $\alpha^{-1}$, which is the time it takes for the autocorrelation to drop by a factor of $e$. This is also often termed the timescale of memory. It is independent from the noise intensity thus solely determined by the dynamics of $x$ through $F(x)$.

An expression for the variance of the linear stochastic process (10) is also easily obtained. To do so it is convenient to write the governing equation in incremental form:

$$
d x=-\alpha x d t+\sigma d B .
$$

The term $d B$ is the Brownian motion also called the Wiener process. Without going into to much technical detail, this can be motivated to be a natural choice: By 
constructing $d B$ as the continuous limit of a discrete process, where small random increments are added. Each small time interval $[t, t+d t]$ is subdivided into $N$ smaller steps, $d t=N \Delta t$. At each of these smaller time steps $t_{n}=t+n \Delta t, n=$ $1, \ldots, N$ a stochastic variable $X_{n}$ is added. $X_{n}$ 's are drawn independently from an unknown distribution with zero mean and a finite variance $\Sigma^{2}$. In the continuum limit, $N \rightarrow \infty$, the central limit theorem ensures that the cumulated noise $\left(X_{1}+\ldots+\right.$ $\left.X_{N}\right)=\tilde{B}(t, d t)$ will be an independent gaussian stochastic noise and by normalising, $d B(t)=\tilde{B}(t, d t) * \sqrt{d t / \Sigma^{2}}$ becomes an independent gaussian noise with $\langle d B\rangle=0$ and $\left\langle d B^{2}\right\rangle=d t$.

From this the variance can be calculated, by noting that the process is stationary, thus:

$$
\begin{aligned}
\left\langle x^{2}\right\rangle & =\left\langle(x+d x)^{2}\right\rangle \\
& \left.=\angle(x-\alpha x d t+\sigma d B)^{2}\right\rangle \\
& =(1-2 \alpha)\left\langle x^{2}\right\rangle+\sigma^{2}\left\langle d B^{2}\right\rangle+\mathcal{O}\left(d t^{2}\right) \Rightarrow \\
\left\langle x^{2}\right\rangle & =\frac{\sigma^{2}}{2 \alpha}
\end{aligned}
$$

This is the fluctuation-dissipation theorem (FDT) connecting the variance of the process $\left\langle x^{2}\right\rangle$ with the noise intensity $\sigma$ and the stability parameter $\alpha$.

For completeness of the analysis of the red noise process, note that the power spectrum is the Fourier transform of the autocorrelation function

$$
\begin{aligned}
P_{x}(\omega) & =2 \int_{0}^{\infty} c_{0} \exp (-\alpha|t|) \cos (\omega t) d t \\
& =c_{0} \int_{0}^{\infty}\{\exp [(-\alpha+\iota \omega) t]+\exp [(-\alpha-\iota \omega) t]\} d t \\
& =c_{0}\left(\frac{1}{\alpha-\iota \omega}+\frac{1}{\alpha+\iota \omega}\right)=c_{0} \frac{2 \alpha}{\omega^{2}+\alpha^{2}}
\end{aligned}
$$

where $\iota$ is the imaginary unit. 

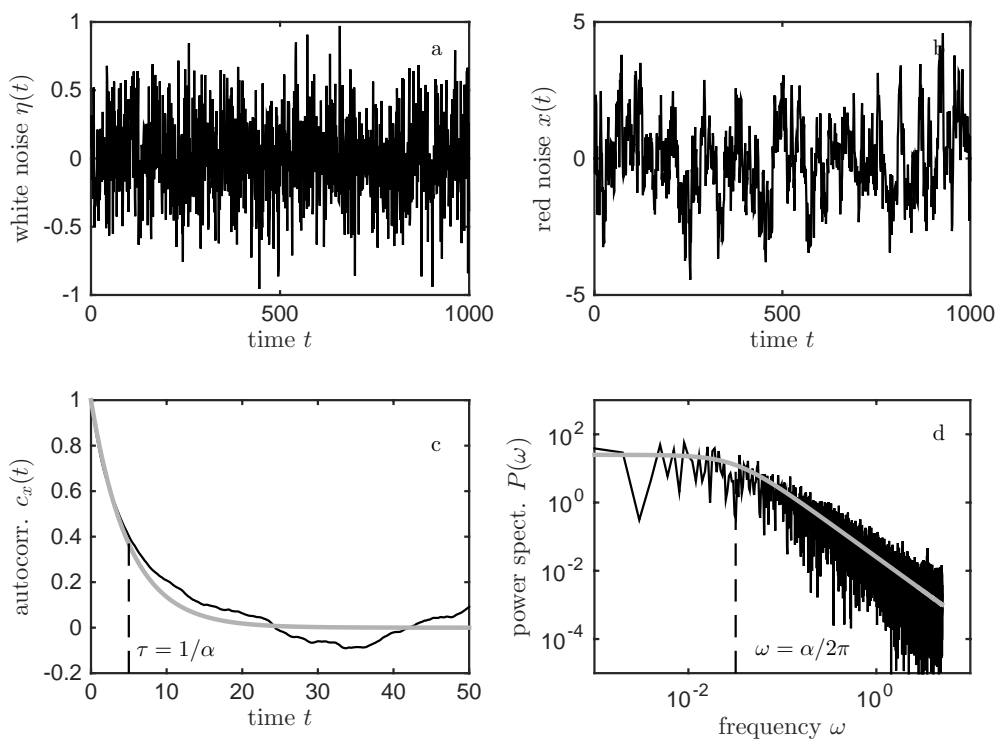

Figure 4: A simulation of the Langevin equation (10). (a) Shows the white noise forcing $\eta$. (b) Shows the climate variable $x$ governed by equation (10). (c) shows the auto-correlation function and $(\mathrm{d})$ the power spectrum for the process. The grey curves are the analytic solutions according to equations (12) and (15). 
The autocorrelation function and the power spectrum are shown in figure 4 . For $\omega \ll \alpha$ we have $P(\omega) \sim 1$, that is, $x$ itself is a white noise signal on timescales long in comparison to the typical timescales of the $x$-dynamics. For $\omega \gg \alpha$ we have $P(\omega) \sim \omega^{-2}$, which is a scaling red noise spectrum.

The simple linear stochastic model captures a fair amount of climatic variability as can be seen from figure 5, where power spectra of an European atmospheric temperature and Atlantic SST anomaly time series are shown. The correlation time in the ocean SST is about two orders of magnitude longer. Thus on the time scale of ocean the atmospheric forcing can be considered as the white noise forcing the ocean SST. This is often phrased as the ocean integrating the atmospheric noise.

As a final remark before returning to detecting tipping points, it is not always a sufficient description of climatic time series to assume fluctuations as due to gaussian white noise. This could be because long term correlations are present in the noise, which will be reflected in a different spectral slope in the power spectrum. One way of modelling this is through fractional brownian motion, where the noise is constructed from requiring specific scaling properties of the autocorrelation (Mandelbrot \& Ness, 1968). It could alternatively be that the noise increments have an extreme distribution, with such a fat tail distribution that the variance is infinite. In this case the central limit theorem is violated. If only moments of the noise variable $\eta$ to the order $\alpha$ are finite, i.e. $\left\langle\eta^{\beta}<\infty\right.$ for $\beta<\alpha<2$ a generalised central limit theorem states that class of distributions, the $\alpha$-stable distributions will be the limiting (attraction) distributions rather than the gaussian when adding increments, as was done when constructing the Brownian noise $d B$. The latter case with $\alpha$-stable noise $d L$ is also called a Levy-process (Uchaikin \& Zolotarev, 1999). Though somewhat exotic, the $\alpha$-stable process does have some relevance for describing climatic time series (Ditlevsen, 1999). 

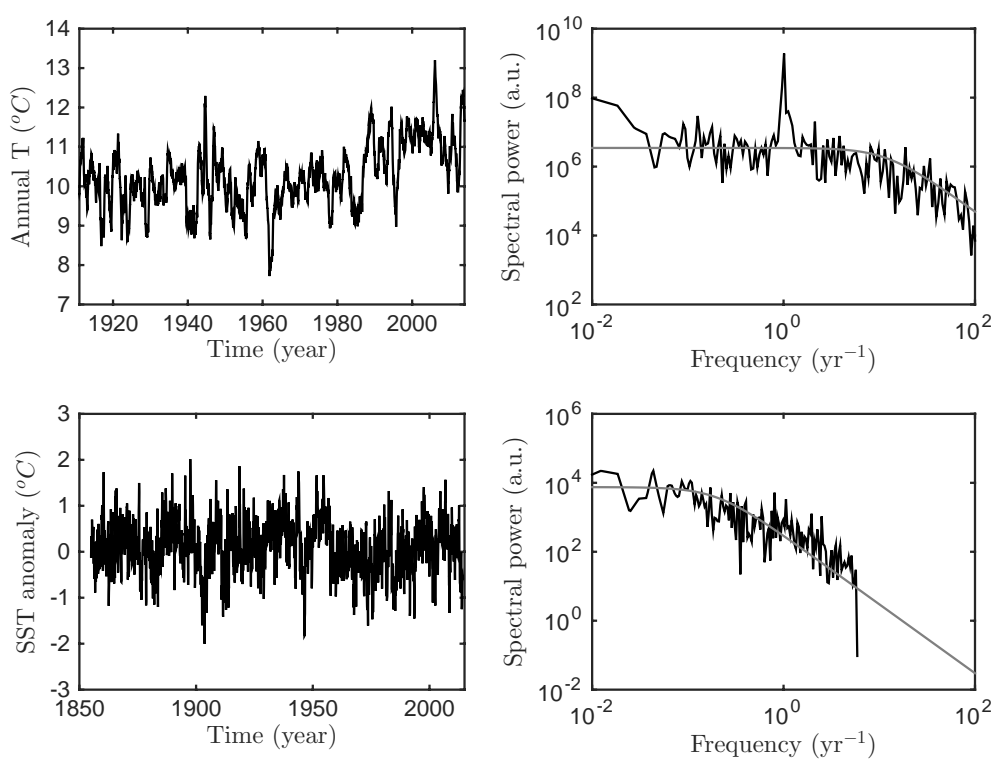

Figure 5: Top panels is the annual averaged surface air temperature measured in Vlissingen (Netherlands) (51.45N, 3.60E) obtained from the KNMI Climate explorer. The power spectrum is for the daily data showing the annual peak on top of the continuous spectrum. The grey curve is the red noise spectrum with correlation time of one month. The bottom panels shows the Atlantic SST anomaly for $(47.5 \mathrm{~N}$, $27.5 \mathrm{~W}$ ) obtained from the updated Kaplan, NCAR record (Kaplan et al., 1998). The grey curve in the power spectrum is a red noise spectrum with correlation time of 5 years. 


\section{Early warning signals for tipping}

Equipped with the essential results for the simple linear stochastic process we can now ask the question if there is a potential for predicting a climatic tipping point before it is actually reached, say through a slow change of the control parameter $\mu$ through a saddle-node bifurcation. For the present climate early warning signals detection is obviously of high societal interest, both in terms of prevention and in terms of mitigation. Another rationale is the attempt to use precursors to abrupt changes observed in the paleoclimatic records to identify underlying mechanisms. This is relevant for understanding the non-linear dynamics of the climate including phenomena like the Dansgaard-Oeschger events, for which we have not yet identified the cause. Consider again the dynamics of the variable $x=x^{0}+\xi$ near the saddlenode bifurcation assuming the additional stochastic noise term as in equation (9). For the noise intensity sufficiently small equation (3) can be expanded linearly around the stable fixed point $x^{0}(\mu)$ (omitting subscript + ):

$$
\dot{\xi}=-(2 \sqrt{-\mu}) \xi+\sigma \eta=-\alpha_{\mu} \xi+\sigma \eta .
$$

Thus the parameter $\alpha_{\mu}=2 \sqrt{-\mu}$ will become small close to the bifurcation point and vanish for $\mu=0$. The autocorrelation time $1 / \alpha_{\mu}$ and the variance $\sigma^{2} /\left(2 \alpha_{\mu}\right)$ will both diverge to infinity at the bifurcation point. Increase in these two statistical quantities are thus potential indicators for an approach to a bifurcation point. The increase in autocorrelation close to the bifurcation point is termed critical slowdown. It reflects the fact that when the drift term $\left(-\alpha_{\mu} \xi\right)$ in equation (16), which pulls the system toward the steady state, becomes small, perturbations away from the steady state can live longer. The mechanical analogy is that of a spring pulling back to the steady state loosing it's strength, thus the phenomenon is also termed softening. The other consequence of softening is that perturbations, caused by the diffusion term $(\sigma \eta)$ will be larger, and thus the variance of the process bigger. The divergence of the variance at the bifurcation point is just a consequence of the disappearance of the drift altogether, where the process becomes a pure diffusion process or Brownian motion, which is not stationary. At this point the linear approximation breaks down and the state will approach some other remote stationary state. 

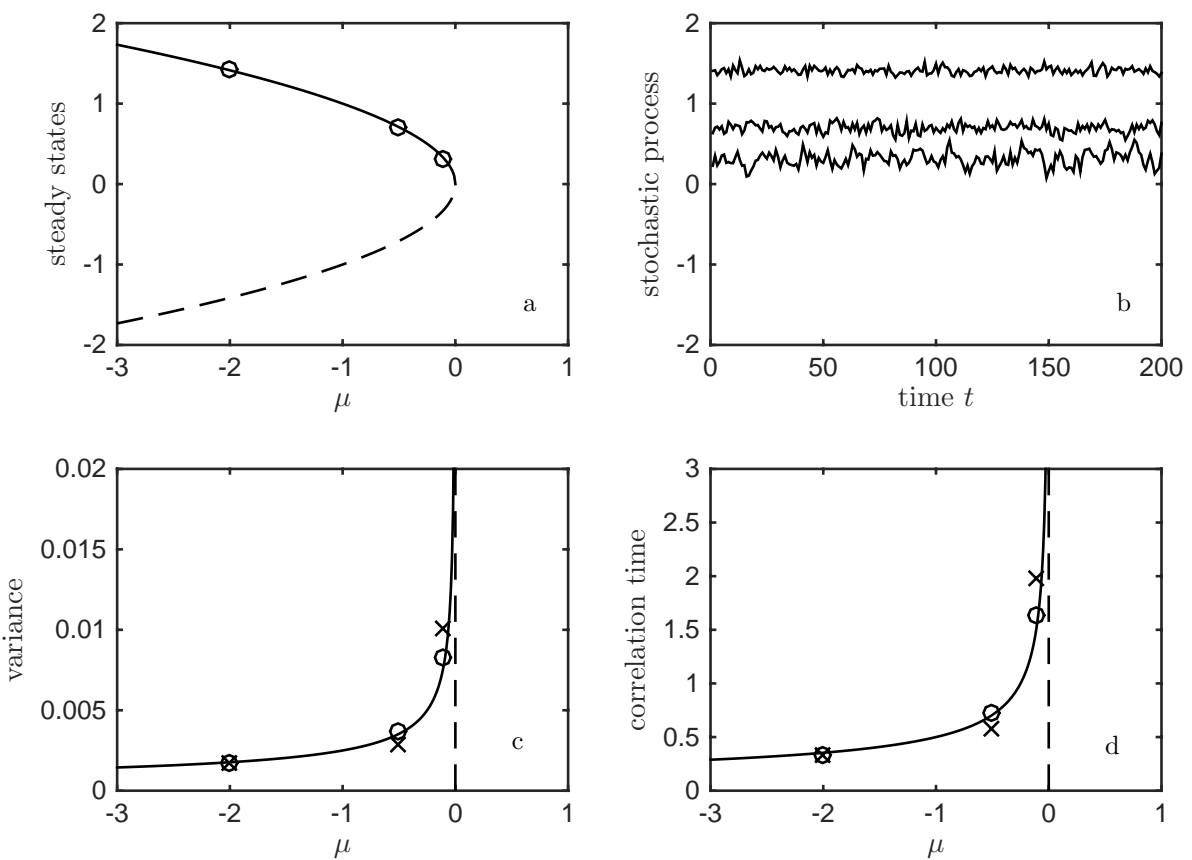

Figure 6: Panel a: The bifurcation diagram for the equation $\dot{x}=-\mu-x^{2}-\mu$. Three different values of the control parameter $\mu$ are indicated by circles in the bifurcation diagram. Panel b: For each of the three values a realisation of the process (9) with $\sigma^{2}=0.1$ are shown; top, middle and bottom curves are for $\mu=-2,-0.5,-0.1$ respectively. Panel c: The variances calculated from the realisations in b are shown by the circles. Crosses are for the linearised processes equation (10) with $\alpha=2 \sqrt{-\mu}$. Panel d: The correlation time calculated from the autocorrelation using (12). Critical slowdown happens as $\mu \rightarrow 0$. The curves in panels $\mathrm{c}$ and $\mathrm{d}$ are the exact results for the linearised process, thus the differences between crosses and the curves indicates the uncertainty in estimating variance and correlation time from the finite realization. The difference between circles and the curve indicates the combined inaccuracy from both linearising and calculating from a finite realisation. 
Obviously, in order for the linear theory to be valid, the change with time in the control parameter $\mu$ must be slow enough that the process can be considered (quasi-)stationary. Furthermore, for issuing an early warning based on a statistical indicator, say, increased variance, a level of confidence must be decided to limit the risks of false positives (warning, when no tipping occurs) and false negatives (missed warning, when a tipping occurs). Assuming that $N$ independent points from the observed process are required for that, such that the $1 / \sqrt{N}$ factor brings the uncertainty in the statistical measure below the accepted level of uncertainty. Then an observation time of the order $N T_{c}$, where $T_{c}$ is the correlation time of the process, is necessary to reliably obtain the indicator. Now take the approach to a saddle-node bifurcation to be described by equation (3). By a change in control parameter close to the critical value $\mu_{c}=0$ given as $\mu(t)=c t, t<0$, the bifurcation point is reached at time $t=0$. For a given $\mu<0$ prior to the bifurcation, the timescale of relative change is $T=(-\dot{\mu} / \mu)^{-1}=-1 / c$, which is also simply the time remaining before reaching the bifurcation point. The correlation time for the quasistationary process is $T_{c}=1 / \alpha_{\mu}=1 /(2 \sqrt{-\mu})$, thus the criterion for reliable early warning is $N T_{c}<T$. The critical slow down, which is $T_{c} \rightarrow \infty$ as $\mu \rightarrow 0$ thus gives a limitation to issuing an early warning before the tipping actually happens. Using the relationships between the control parameter and the time scales, the criterion above implies $c<2 \sqrt{-\mu} / N$. Thus the closer to the bifurcation point the system is (the closer to zero $\mu$ is), the slower should the change in $\mu$ be (the smaller $c$ ) in order to be able to warn ahead of time.

Before investigating the implications of the slow down for possibilities of early warning, a third precursor to a bifurcation beside the increases in autocorrelation and variance is presented here: An increased skewness in the quasi-stationary distribution of the process is in principle also a precursor (Guttal \& Jayaprakash, 2008), which goes beyond the linear theory. Referring again to the effectively one dimensional dynamics along the most unstable direction near the bifurcation point, the dynamics (9) can, with $U_{\mu}(x)=-\int^{x} F_{\mu}(x) d x$, conveniently be described as a potential flow with a stochastic noise,

$$
\dot{x}=-\frac{d U_{\mu}}{d x}+\sigma \eta
$$

A simple multi-state dynamics is described through the double well potential

$$
U_{\mu}(x)=\frac{x^{4}}{4}-\frac{x^{2}}{2}+\mu x
$$

In this case a set of saddle-node bifurcations occur for $\mu_{ \pm}= \pm 2 \sqrt{3} / 9$. When ap- 
proaching one of the bifurcation points, say $x_{0}\left(\mu_{+}\right)=(\sqrt{3} / 9)^{1 / 3}$, the rightmost steady state will merge with the middle unstable steady state. The potential becomes increasingly asymmetric with respect to perturbations away from the steady state in the vicinity of $x_{0}(\mu)$ as $\mu \rightarrow \mu_{+}$. Thus the drift in the direction of the other steady state will be larger than the drift away from the other steady state, thus the distribution becomes skewed. 

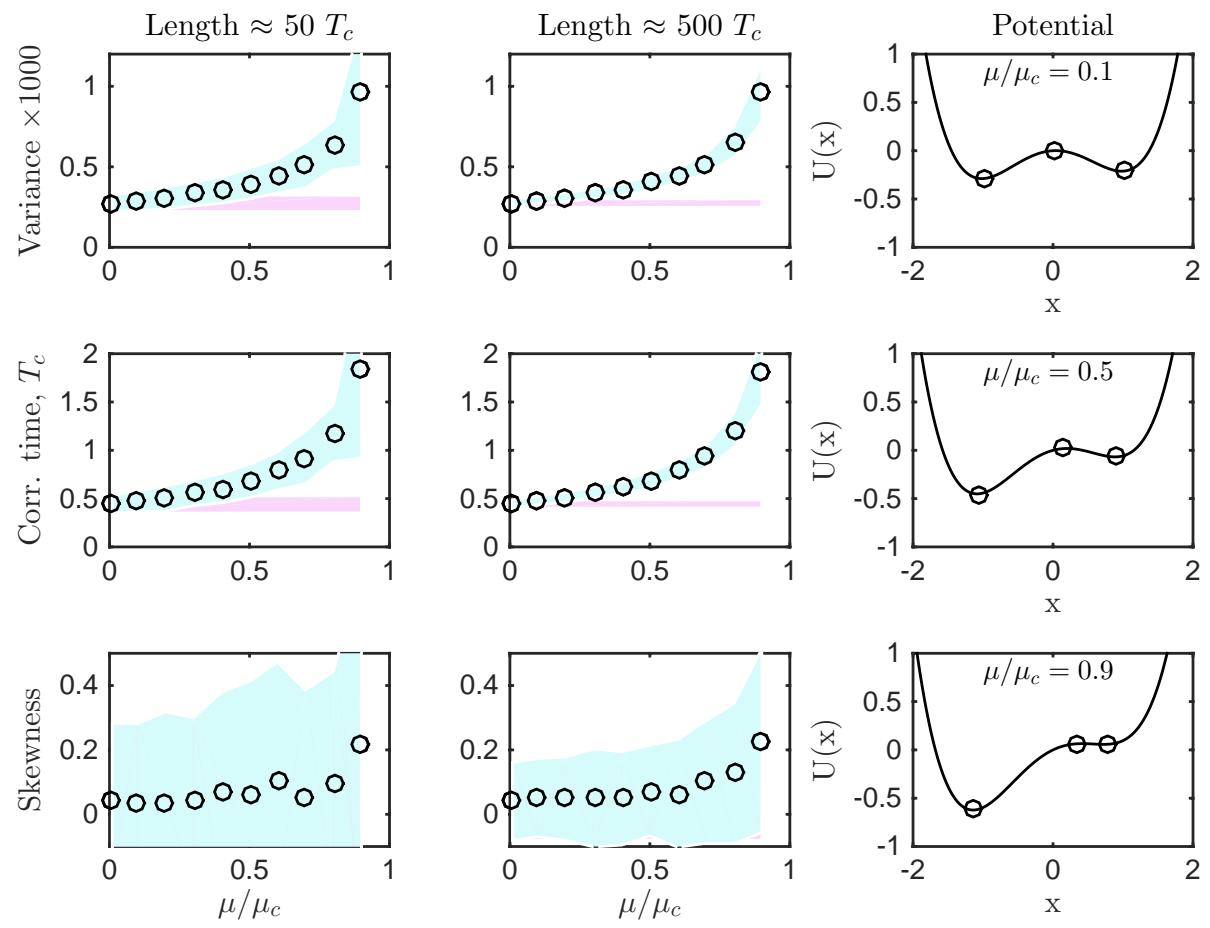

Figure 7: A set of realisations of the process (17) with $0 \leq \mu / \mu_{c} \leq 0.9$ have been simulated. For each value of $\mu$ and for each of 100 realisations of 50 time units length (first column) and 500 time units (second column) variance, correlation time and skewness have been calculated. The means are indicated in open circles, while the light blue area indicates the 2-sigma confidence interval. The horizontal purple bars indicate the 2-sigma confidence interval for $\mu / \mu_{c}=0$. The third column shows the shapes of the potential for $\mu / \mu_{0}=0.1,0.5$ and 0.9 . The steady states are indicated by the circles. 
The effect of critical slowdown on early warning is illustrated in figure 7 . The process (17) with $0 \leq \mu \leq 0.9 \mu_{0}$ has been simulated. For each value of $\mu$, represented by the open circles in the left columns, 100 realisations have been performed. In the first column the processes are 50 time units long, while in the middle column the processes are 500 time units long. In the three rows the tipping point indicators, variance, autocorrelation, represented by the correlation time $T_{c}$ and the skewness are shown. The correlation time is obtained from fitting the autocorrelation function to the best fit exponential according to equation (12). From the middle panels it is seen that the length of the records corresponds to approximately 50 and 500 times the correlation time, less as $\mu / \mu_{c}$ approaches 1 . The means of the indicators over the 100 realisations are plotted as open circles. The filled blue areas represent the 2-sigma uncertainty obtained from the 100 realisations. The purple areas are the corresponding 2-sigma uncertainty for the constant value $\mu=0$. We can now consider the scenario that the control parameter $\mu$ is approaching $\mu_{c}$ linearly in time, $\mu(t)=c t+\mu_{0}$ for $t<0$, reaching the bifurcation point at $t=0$. The question is then how close to the critical value must $\mu$ be before an early warning can be issued: In order to avoid false positives (predicting a bifurcation when non is about to occur) at the 2-sigma level the indicator must fall in the blue area not overlapping the purple area, likewise in order to avoid false negatives (missing a warning for a bifurcation, which is about to happen) at the 2-sigma level the indicator must fall in the purple area, not overlapping the blue area. From figure 7 it is seen that for this process skewness is not a good early warning indicator, while both variance and correlation time can be used. In the left column the early warning can be issued when $\mu \approx 0.6 \mu_{0}$, while in the middle column case the early warning can be issued when $\mu \approx 0.4 \mu_{0}$.

\section{Noise induced tipping}

A transition from one stable equilibrium to another can happen without a bifurcation if the noise by chance brings the state across the potential barriere. This is termed noise induced tipping (n-tipping) in contrast to the bifurcation induced tipping (btipping). 


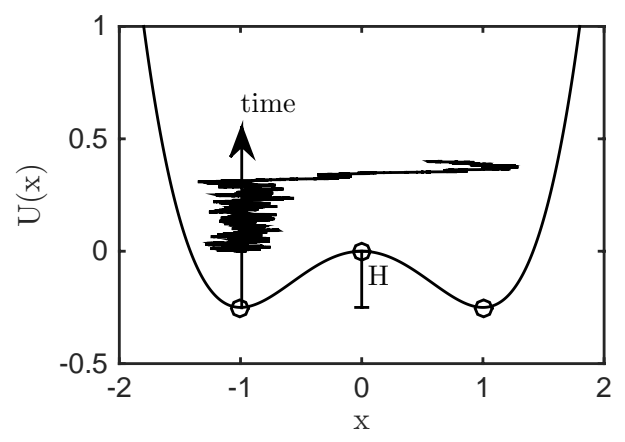

Figure 8: A realisation of the process (17), which crosses the potential barrier of height $H$. The circles indicate the steady states, where the one on top of the barrier is unstable.

In figure 8 this is illustrated by a realisation of the process described by equations (17) and (18) for $\mu=0$. In this case there are no early warning signals prior to the tipping, since the potential does not change in time. The waiting time for a tipping, or a jump to the other stable equilibrium depends on the height $H$ of the potential barrier separating the two states and on the variance of the noise $\sigma^{2}$ kicking the system away from equilibrium. This is the usual Arrhenius time for escape $T \sim \exp \left(H / \sigma^{2}\right)$. We shall not go into mathematical details, just give a heuristic argument for this in the case $\sigma^{2} \ll H$, which will of course only hold sufficiently far away from a bifurcation point. From the fluctuation-dissipation theorem for the linear theory (14) we can estimate the mean square fluctuation within a correlation time as $\left\langle x^{2}\right\rangle \sim \sigma^{2} T_{c}$. In order for a noise induced transition to happen $n$ independent increments of the order $\Delta x=\sqrt{\left\langle x^{2}\right\rangle}$ must all be in the same "uphill" direction. In this time the drift towards the equilibrium is of the order $\Delta x \sim(\Delta U / \Delta x)\left(n T_{c}\right)$, thus $(\Delta x)^{2} \sim \Delta U\left(n T_{c}\right)$. Thus to overcome a barrier of height $\Delta U=H$, the number of independent noise increments can be estimated from $(\Delta x)^{2} \sim \sigma^{2} T_{c} \sim H n T_{c} \Rightarrow$ $n \sim H / \sigma^{2}$. Thus with probability $p$ for an noise increment towards the barrier, the probability for $n$ increments in that direction is $p^{n}=\exp (n \log p)$, thus with $\log p$ of the order -1 , the waiting time is of the order $T \sim 1 / \exp (-n)=\exp \left(H / \sigma^{2}\right)$.

In the situation considered in the previous section with a bifurcation approached in time, $\mu(t)=c t+\mu_{0}$, the system will with very high probability jump away from the equilibrium state prior to the time of the bifurcation $(t=0)$. This typically happens when $T \sim \exp \left(H(t) / \sigma^{2}\right)$ becomes smaller than $T_{c}$ as $H(t) \rightarrow 0$ at the bifurcation point. Thus only in the limit $\sigma \rightarrow 0$ a pure b-tipping will occur. 


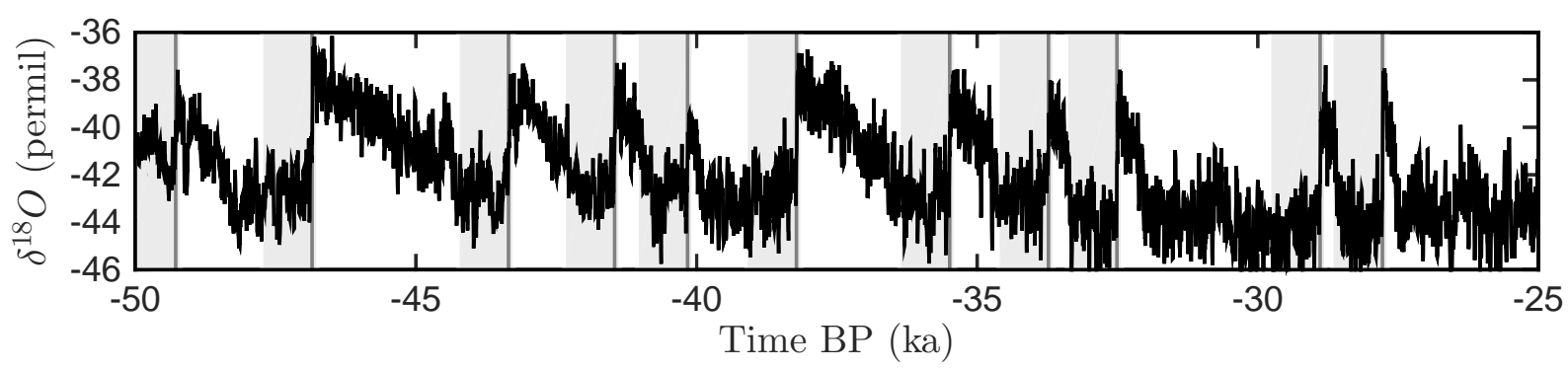

Figure 9: A section of the NGRIP ice core isotope record showing the abrupt DO events. The vertical lines are the jumps, while the grey areas to the left of the lines indicate 900 ka prior to the jumps.

\section{Identifying tipping mechanism through early warn- ing signals}

The paleoclimatic record documents abrupt climate changes, which are often of unknown origin. Most pronounced are the Dansgaard-Oeschger (DO) events (Dansgaard et al., 1993) during the last glacial period. These are abrupt jumps from the cold glacial climate, the stadials, to warm interstadial states on millennial time scale with gradual cooling until a jump back to the cold glacial climate occurs.

Different mechanisms have been proposed for the DO events: A reduction in the freshwater input into the North Atlantic could trigger a shift of the meridional overturning circulation (MOC) to the on-mode, leading to a strong northern warming (Clark et al., 2002). The evidence from Heinrich events of massive iceberg discharge suggests the stability of the ice sheet to be important (Bond \& Lotti, 1995). Alternatively, a fast retreat of North Atlantic sea ice leading to a warming through the ice albedo feedback mechanism (Gildor \& Tziperman, 2003). An other proposed mechanism is a fast-slow cycle of ice shelf breakup combined with sea ice retreat, rapid warming followed by a slow rebuild of ice shelves (Petersen et al., 2013). Some of these proposed mechanisms involve the possibility of a bifurcation triggered by an external change of a control parameter. A millennium time scale variation in the solar flux has been proposed (Braun et al., 2005). Alternatively, the jumping between the two steady climate states could be noise induced without any external change in parameters. We are thus asking if the DO events are most likely to be due to a b-tipping or an n-tipping scenario (Ditlevsen \& Johnsen, 2010). The question is then if the climate record contains information which would discriminate between the two scenarios. This is exactly what the early warning signals do when assuming 
the two different scenarios governed by equation (17). Firstly, the b-tipping with $\mu=\mu_{c} t / 900$, where $\mu_{c}$ is the critical value where the bifurcation occurs. This means that at time $t=0$ the potential (18) is symmetric, and slowly changing such that at time $t=900$ the bifurcation occurs. First column in Figure 9 shows a realisation of this process, where the early warning signals are calculated in windows indicated by the bar in the middle panel. A clear increase in variance and a somewhat less clear increase in autocorrelation are seen prior to the actual jump, which happens by a noise induced jumping of a small barrier a short time before $t=900$. It should be noted that in the presence of any noise the escape in the b-tipping scenario will typically happen at some time prior to reaching the bifurcation point, where the barrier for escape is comparable with the noise level.

Secondly, the n-tipping scenario is shown in the second column as a realisation of the process with constant $\mu=0$. The simulation is run long enough for a jump to happen. Time is then a posterior reset, such that the n-tipping occurs at $t=900$. From the two lower panels it is seen that there are no early warning signals prior to the n-tipping. In the same way the third column shows the DO events observed in the NGRIP ice core all aligned such that the jump from the stadial state to the interstadial state occurs at $t=900 \mathrm{ka}$. The variance and autocorrelation are calculated in the same way as for the simulations and show no sign of increase prior to the jump. We can thus exclude the b-tipping scenario and favour the ntipping scenario for the DO events. This finding implies that abrupt climate events of DO type has very limited predictability for two reasons. Firstly, there are no early warnings, thus the predictability is essentially the same as the predictability of the noise, which is of the order of predictability of perhaps El Nino type variations or even shorter time scale chaotic weather variations. Secondly, a slowly varying parameter $\mu$ controlling the stability could in principle be predictable had it, say, a periodic astronomical origin (Braun et al., 2005). The recurrence time for DO events has been subject for some debate, since it was noted that there was apparently a preferred waiting time between events of a multiple of 1470 years (Schulz, 2002). It was later shown that the waiting time statistics for the DO events is comparable with an exponential waiting time distribution (Ditlevsen et al., 2005; Ditlevsen et al., 2007). Thus the apparent periodicity is by pure chance. The n-tipping scenario gives further support to this finding. 

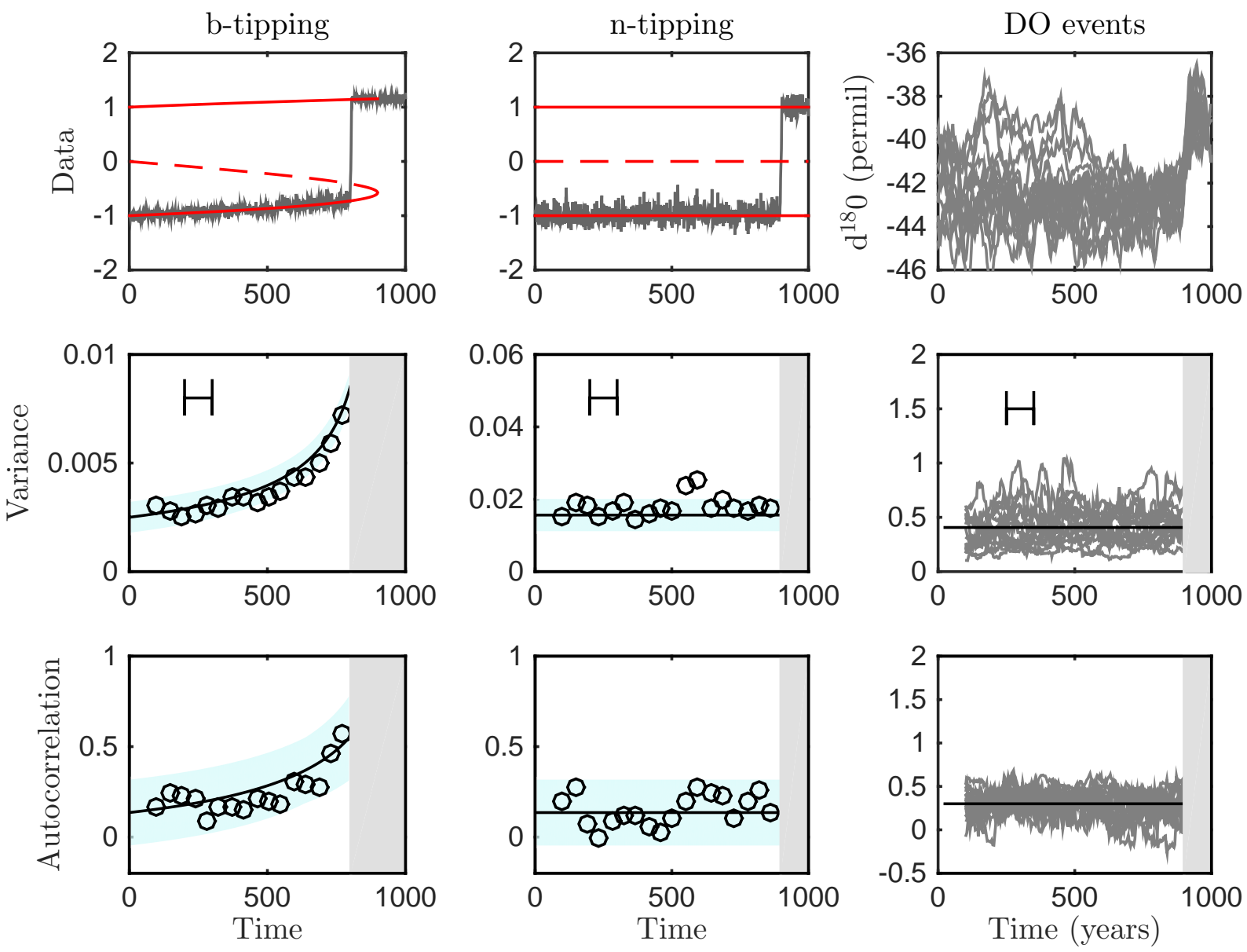

Figure 10: The first column shows a realisation of the b-tipping scenario, governed by equations (17) and (18) where the control parameter change in time as $\mu=\mu_{c} t / 900$. The red lines are the steady states, and the bifurcation happens at $t=900$ (arbitrary units). The lower panels show variance and autocorrelation calculated within a running window of 100 time units indicated by the bar in the middle panel. Both early warning signals increase prior to the tipping. In the second column an n-tipping scenario is shown. The red lines indicates that the steady states do not change in time. Time is reset, such that an n-tipping happens at $t=900$. The variance and autocorrelation do not increase prior to the jump, thus there are no early warnings prior to the n-tipping. The light blue bands are obtained from a set of 100 realisations as the 2-sigma confidence level. The last column shows the DO events in the NGRIP ice core (sections in figure 9 marked in grey) lined up such that the jumping happens at $t=900 \mathrm{ka}$ (red lines in figure 9 ). The $\$ 20$ events do not show increase in variance or autocorrelation prior to the jump, indicating that they are n-tipping events. 


\section{References}

Berger, A. 1978. Long-term variations of daily insolation and Quaternary climatic change. J. Atmos. Sci., 35, 2362-2367.

Bond, G. C., \& Lotti, R. 1995. Iceberg discharges into the North-atlantic on millennial time scales during the last glaciation. Science, 267, 1005-1010.

Braun, H., Christi, M., Rahmstorf, S., Ganopolski, A., Mangini, A., Kubatski, C., Roth, K., \& Kromer, B. 2005. Possible solar origin of the 1,470-year glacial climate cycle demonstrated in a coupled model. Nature, 438, 208-211.

Budyko, M. I. 1969. The effect of solar ratiation variations on the climate of the Earth. Tellus, 21, 611-619.

Clark, P. U., Pisias, N. G., Stocker, T. F., \& Weaver, A. J. 2002. The role of the thermohaline circulation in abrupt climate change. Nature, 415, 863869.

Dansgaard, W., Johnsen, S. J., Clausen, H. B., Dahl-Jensen, D., Gundestrup, N. S., Hammer, C. U., Hvidberg, C. S., Steffensen, J. P., Sveinbjornsdottir, A. E., Jouzel, J., \& Bond, G. 1993. Evidence for general instability of past climate from a 250-kyr ice-core record. Nature, 364, 218-220.

Ditlevsen, P. D. 1999. Observation of alpha-stable noise and a bistable climate potential in an ice-core record. Geophys. Res. Lett., 26, 1441-1444.

Ditlevsen, P. D., \& Johnsen, S. 2010. Tipping points: Early warning and wishful thinking. Geophys. Res. Lett., 37, L19703.

Ditlevsen, P. D., Kristensen, M. S., \& Andersen, K. K. 2005. The recurrence time of Dansgaard-Oeschger events and limits on the possible periodic component. $J$. Climate, 18, 2594-2603.

Ditlevsen, P. D., Andersen, K. K., \& Svensson, A. 2007. The DO-climate events are probably noise induced: statistical investigation of the claimed 1470 years cycle. Climate of the Past, 3, 129-134.

Gardiner, C.W. 1985. Handbook of Stochastic Methods. Springer Verlag, N.Y.

Gildor, H., \& Tziperman, E. 2003. Sea-ice switches and abrupt climate change. Phil. Trans. R. Soc. Lond. A, 361, 1935-1944. 
Guttal, Vishwesha, \& Jayaprakash, Ciriyam. 2008. Changing skewness: an early warning signal of regime shifts in ecosystems. Ecology Letters, 11(5), 450-460.

Hasselmann, K. 1976. Stochastic Climate Models. Tellus, 28, 473-485.

Hays, J., Imbrie, J., \& Shackleton, N. 1976. Variations in earths's orbit: Pacemaker of the ice ages. Science, 194, 1121-1132.

Hoffman, P. F., Kaufman, A. J., Halverson, G. P., \& Schrag, D. P. 1998. A Neoproterozoic Snowball Earth. Science, 281, 1342-1346.

Kaplan, A., Cane, M., Kushnir, Y., Clement, A., Blumenthal, M., \& Rajagopalan, B. 1998. Analyses of global sea surface temperature 1856-1991. Journal of Geophysical Research, 103, 18,567-18,589.

Kleidon, Axel, Fraedrich, Klaus, \& Heimann, Martin. 2000. A Green Planet Versus a Desert World: Estimating the Maximum Effect of Vegetation on the Land Surface Climate. Climatic Change, 44, 471-493.

Lenton, T. M., Held, H., Kriegler, E., Hall, J. W., Lucht, W., Rahmstorf, S., \& Schellnhuber, H. J. 2008. Tipping elements in the Earth's climate system. Proceedings of the National Academy of Sciences, 105, 1786-1793.

Lisiecki, L. E., \& Raymo, M. E. 2005. A Pliocene-Pleistocene stack of 57 globally distributed benthic D18O records. Paleoceanography, 20, PA1003.

Ma, T, \& Wang, S. 2005. Bifurcation Theory and Applications, Series on Nonlinear Science Series A: Volume 53. World Scientific.

Majda, Andrew J., Timofeyev, Ilya, \& Vanden-Eijnden, Eric. 2003. Systematic Strategies for Stochastic Mode Reduction in Climate. Journal of the Atmospheric Sciences, 1705-1722.

Mandelbrot, Benoit B., \& Ness, John W. Van. 1968. Fractional Brownian Motions, Fractional Noises and Applications. SIAM Review, 10(4), 422-437.

Milankovitch, M. 1930. Mathematiche Klimalehre und Astronomiche Theorie der Klimaschwankungen, Handbuch der Klimalogie Band 1 Teil A. Borntrager Berlin.

North GRIP members. 2004. High resolution Climate Record of the Northern Hemisphere reaching into the last Glacial Interglacial Period. Nature, 431, 147-151. 
Paillard, D. 1998. The timing of Pleistocene glaciations from a simple multiple-state climate model. Nature, 391, 378-381.

Papanicolaou, G. C., \& Kohler, W. 1974. Asymptotic theory of mixing stochastic ordinary differential equations. Communications on Pure and Applied Mathematics, 27, 641-668.

Petersen, S. V., Schrag, D. P., \& Clark, P. U. 2013. A new mechanism for DansgaardOeschger cycles. Paleoceanography, 28, 24-30.

Rahmstorf, S. 1995. Bifurcations of the Atlantic thermohaline circulation in response to changes in the hydrological cycle. Nature, 378, 145-149.

Schulz, M. 2002. On the 1470-year pacing of Dansgaard-Oeschger warm events. Paleoceanography, 17, 1029/200PA000571.

Sellers, W. D. 1969. A Global Climatic Model Based on the Energy Balance of the Earth-Atmosphere System. J. Applied Meteorology, 8, 392-400.

Stommel, H. 1961. Thermohaline convection with two stable regimes of flow. Tellus, 13, 224-230.

Uchaikin, Vladimir V., \& Zolotarev, Vladimir M. 1999. Chance and Stability: Stable Distributions and their Applications. De Gruyter.

Weertman, J. 1961. Stability of ice-age ice sheets. Journal of Geophysical Research, 66, 3783-3792. 


\section{Index}

Arrhenius escape time, 23

autocorrelation, 12

autocorrelation function, 12

b-tipping, 1, 22

bifurcation induced tipping, 22

bifurcation theory, 4

control parameter, 2

critical slowdown, 17

Dansgaard-Oeschger events, 2, 24

early warning signals, 17

Earth system models, 9

energy balance model, 7

feedback factor, 4

fluctuation-dissipation theorem, 13

ice core record, 2

Langevin equation, 11

n-tipping, 1, 22

NGRIP ice core, 24

noise induced tipping, 22

ocean sediment record, 2

Ornstein-Uhlenbeck process, 11

potential flow, 19

power spectrum, 13

red noise, 15

red noise process, 11

saddle-node bifurcation, 11

skewness, 19

stochastic climate model, 11

stochastic dynamics, 11

time scale separation, 10

tipping point, 1 